\title{
GERMINAÇÃO DE SEMENTES DE ASTERACEAE NATIVAS NO RIO GRANDE DO SUL, BRASIL ${ }^{1}$
}

\author{
Alfredo Gui Ferreira ${ }^{2}$ \\ Bibiana $\mathrm{Cassol}^{3}$ \\ Shirley Galli Taylor da Rosa ${ }^{4}$ \\ Tânia Sales da Silveira ${ }^{4}$ \\ Ana Lúcia Stival ${ }^{4}$ \\ Adriana Andreoli Silva ${ }^{5}$
}

Recebido em 15/08/00. Aceito em 26/04/01

\begin{abstract}
RESUMO - ( Germinação de sementes de Asteraceae nativas no Rio Grande do Sul, Brasil)
Aquênios (sementes) recém coletados, de treze espécies nativas de Asteraceae comuns nos ambientes abertos da região sul do Brasil foram testados quanto à germinação em temperaturas alternadas $\left(20 / 10 ; 25 / 15 ; 30 / 20 ; 35 / 25^{\circ} \mathrm{C}\right)$ e sob temperaturas constantes $\left(20 ; 25 \mathrm{e} 30^{\circ} \mathrm{C}\right) \mathrm{com}$ ou sem luz. A temperatura ótima para germinação varia entre as espécies, sendo que as espécies Elephantopus mobilis; Eupatorium laevigatum; Mikania cordifolia; Senecio oxyphyllus; Trixis prastens germinam de forma semelhante em todas temperaturas testadas. Eclipta alba tem sua germinação promovida a $30^{\circ} \mathrm{C}$. Tagetes minuta tem a germinação das sementes promovida a $20^{\circ} \mathrm{C} . \mathrm{Em}$ Senecio heterotrichius; $\mathrm{S}$. selloi; Stenachaenium campestre; Symphyopappus casarettoi e Vernonia nudiflora as sementes germinam igualmente a 20 ou $25^{\circ} \mathrm{C}$.. A luz promoveu a germinação de todas espécies exceto para Stenachaenium campestre e Tagetes minuta, sendo esta última espécie fotoblástica negativa. Quanto ao tempo médio de germinação, as espécies podem ser divididas em ; rápidas- menos de 5 dias (Baccharis trimera; Eclipta alba; Elephantopus mollis; Stenachaenium campestre e Vernonia nudiflora); intermediárias: entre 5 e 10 dias ( Eupatorium laevigatum; Mikania cordifolia e Tagetes minuta) ; lentas: mais de 10 dias (Senecio heterotrichius; S.oxyphyllus; S. selloi; Symphyopappus casaretto e Trixis praestans). Os resultados mostram que a germinação de sementes de Asteraceas variam com a temperatura e o regime de luz; podendo prover uma base inicial para interpretação de efeitos sazonais sobre a germinação e estabelecimento a campo. Em adição, comentários sobre o substrato ágar ou areia são feitos.
\end{abstract}

Palavras-chave-Germinação, temperatura, luz, Compostas, Senecio, Baccharis, Eclipta, Elephantopus, Eupatorium, Mikania, Stenachenium, Sumphyopappus, Tagetes, Trixis.

\begin{abstract}
Germination of seeds of Asteraceae natives of Rio Grande do Sul, Brazil).
Achenes of thirteen native Asteraceae species common to the natural grassland or weeds of the southern region of Brazil were tested for germination over a range of alternating temperatures $\left(20 / 10 ; 25 / 15 ; 30 / 20\right.$ and $\left.35 / 25^{\circ} \mathrm{C}\right)$, and under constant temperatures $\left(20 ; 25\right.$ and $\left.30^{\circ} \mathrm{C}\right)$ with light or not .Only fresh collected achenes (herein $=$ seeds) were used. The optimum temperature for germination differed among the species, with Elephantopus mobillis; Eupatorium
\end{abstract}

\footnotetext{
${ }^{1}$ Departamento de Botânica / Universidade Federal do Rio Grande do Sul / Av..Bento Gonçalves 9500 -Prédio 43423 - Porto Alegre, RS, Brasil - CEP 91509-900

${ }^{2}$ Professor Titular - Bolsista do CNPq

${ }^{3}$ Mestre em Botânica - UFRGS

${ }^{4}$ Doutoranda em Botânica - UFRGS

${ }^{5}$ Bióloga
} 
laevigatum; Mikania cordifolia; Senecio oxyphyllus; Trixis praestans germinating the most over all temperatures tested. Eclipta alba seeds germination was promoted at $30^{\circ} \mathrm{C}$. Colder treatments promoted germination in Tagetes minuta, and in Senecio heterotrichius; S.selloi; Stenachaenium campestre; Symphyopappus casarettoi and Vernonia nudiflora germination was equivalent at 20 or $25^{\circ} \mathrm{C}$. Light promoted germination for all species except in Stenachaenium campestre and Tagetes minuta, the latter being a negative photoblastic species. According to the mean time for germination, the species could be ranked in: fast -less than 5 days- (Baccharis trimera; Eclipta alba; Elephantopus mollis; Stenachaenium campestre and Vernonia nudiflora); intermediate: between 5 and 10 days- (Eupatorium laevigatum; Mikania cordifolia and Tagetes minuta) ; slow: more than 10 days-(Senecio heterotrichius; S.oxyphyllus; S.selloi; Symphyopappus casarettoi; Trixis praestans). The results show that germination of seeds of a range of Asteraceae species varies with temperature and light regime; they provide an initial basis on which to test and interpret the effects of seasonal factors on germination and field establishment. In addition, comments on the agar and sand substrates were made.

Key words - Germination, temperature, light, Compositae, Senecio, Baccharis, Eclipta, Elephantopus, Eupatorium, Mikania, Stenachaenium, Symphyopappus, Tagetes, Trixis.

\section{Introdução}

A família Asteraceae é uma das mais representativas no ambiente campestre do sul do Brasil (Boldrini \& Eggers, 1996), sendo que muitas espécies desta família são ruderais e/ou invasoras de pastagens (Aranha et al. 1987: Gavilanes \& Angieri-Filho, 1991; Kissmann \& Groth, 1992), comportando-se como plantas agressivas na sua instalação , especialmente na ocupação de áreas perturbadas. A agressividade pode se manifestar por produção de substâncias químicas liberadas no meio que inibam ou retardem a germinação e/ou estabelecimento de competidoras (Cheng, 1992) ou ainda por rápida germinação dos diásporos e estabelecimento da planta. As sementes germinarão, quando as condições forem favoráveis não somente para germinação, mas também para o desenvolvimento e crescimento da futura plantinha (Egley, 1995).

Luz, ausência ou presença, e sua qualidade, temperatura e suas alternâncias, concentração de gases e água em torno dos diásporos são fatores ambientais mais comuns que regulam a germinação (Schupp \& Fuentes, 1995; Ghersa et al. 1992; Baskin \& Baskin, 1998). É comum haver interação entre os fatores acima mencionados, sendo a interação entre luz e temperatura das mais importantes (Taylorson \& Hendricks,
1972; Frankland \& Taylorson, 1983), tanto quanto esta temperatura é constante (Toole et al., 1956; Toledo et al., 1990) quanto seja ela flutuante ou alternada ( Thompson, 1974; Esashi \& Tsukada, 1978: Thompson \& Grime, 1983).

A presença de papus nos frutos de um número significativo de espécies, nesta família , garante um mecanismo eficiente de dispersão dos propágulos a áreas distantes. Nos locais abertos onde o vento pode fluir mais livre, é o ambiente especialmente propício para dispersão de tais tipos de propágulos, que podem permanecer junto à superfície do solo por tempo variável. Nestes ambientes, a insolação, e portanto a luz, é abundante. Nos campos e ambientes abertos seja pelo manejo com animais, gado, seja pela remoção da vegetação para cultivos, constantemente há retirada, ainda que parcial, das plantas que cobrem o solo. Isto propicia que haja variação de exposição mais intensa à energia radiante tanto em temperatura como luz. A luz é normalmente filtrada através das folhas, tornando-se mais pobre em vermelho $(660 \mathrm{~nm})$ e portanto com níveis mais altos de vermelho extremo (730nm) (Frankland \& Taylorson, 1983). Estes são os comprimentos de onda nos quais há reversão do fitocromo tido como o principal receptor da luz que influi no mecanismo de germinação das sementes fotoblásticas (Kendrick, 1976; Smith \& Morgan, 1983). 
Com o objetivo de verificar o comportamento germinativo dos diásporos de Asteraceae de ambientes campestre ou ruderal, bem como de plantas destes ambientes de interesse medicinal, é que este estudo foi realizado.

\section{Materiais e métodos}

Foram usados como materiais experimentais, aquênios de treze espécies da família Asteraceae (Compositae) ocorrentes no estado do Rio Grande do Sul, Brasil: Baccharis trimera (Less)DC, Eclipta alba Hassk.; Elephantopus mollis H.B.\& K.; Eupatorium laevigatum Lam.; Mikania cordifolia Willd.; Senecio heterotrichius DC., S. oxyphyllus DC.; S.selloi DC.; Stenachaenium campestre Baker; Symphyopappus casarettoi B.L.Rob.; Tagetes minuta L.; Trixis praestens (Vell.)Cabrera e Vernonia nudiflora Less.. Os capítulos, logo após a coleta, eram deixados por alguns dias para secar sobre a bancada no laboratório à temperatura ambiente ( $18 \mathrm{a}$ $22^{\circ} \mathrm{C}$ ) sendo então extraídos os aquênios, daqui por diante usados como sinônimo de sementes.

As sementes assim obtidas, eram selecionadas com o uso de uma lupa de mão, separando-se as visivelmente mal formadas, aquênio translúcidos. Em alguns casos, o papus foi cortado com uma tesoura, sendo a seguir imersos por dez segundos em etanol $70 \%$, para diminuir a tensão superficial, seguindo-se uma desinfestação com solução de hipoclorito de sódio com cerca de $1 \%$ de cloro nascente. Logo a seguir as sementes foram lavadas com água estéril.

Cinqüenta ou trinta e cinco sementes, assim tratadas, foram colocadas em placas de Petri de $9 \mathrm{~cm}$ de diâmetro com 3 repetições por tratamento. Foram adicionados às placas $30 \mathrm{ml}$ de solução geleificada com agar a $1 \%$ estéril as quais foram colocadas nas temperaturas apropriadas em câmaras de germina- ção tipo DBO-FANEN. As temperaturas usadas para os testes foram constantes de $20 ; 25$ e $30^{\circ} \mathrm{C}$, com luminosidade de cerca de $4 \mathrm{Wm}^{-2}$, com fotoperiodo de 12 ou 8 horas de luz, fornecidas por três lâmpadas fluorescentes e uma grolux. O escuro era obtido pelo envolvimento das placas em laminado de alumínio flexível. Em alguns experimentos usouse temperaturas de $35^{\circ} \mathrm{C}$, com luz, ou 10 e $15^{\circ} \mathrm{C}$, neste caso no escuro. Foram utilizadas também temperaturas alternadas de $: 35-25^{\circ} \mathrm{C}$; $30-20^{\circ} \mathrm{C}: 25-15^{\circ} \mathrm{C}$ e $20-10^{\circ} \mathrm{C}$, sendo a mais alta com luz por oito horas diárias e a mais baixa no escuro. Diariamente pela manhã e fim da tarde, as placas eram transferidas para a temperatura apropriada. Nos tratamentos na luz, houve acompanhamento diário a partir do segundo dia até quinze dias da semeadura. Para as espécies medicinais E.alba; E.laevigatum; S.campestre e T.minuta este acompanhamento foi por 30 dias. Nos tratamentos no escuro, foi somente feita uma contagem ao final do tratamento. Nas espécies tidas como medicinais anteriormente destacadas, houve leitura diária, usando luz verde de segurança (Labouriau \& Costa, 1976). A emergência da radícula, numa extensão de mais da metade do aquênio, foi o critério usado para germinação. Os dados obtidos foram analisados pelo teste Bartlett para verificação da homogeneidade (Sokal \& Rohlf, 1981) e seguidos de ANOVA e de DUNCAN a 5\% (Little \& Jackson, 1978). O tempo médio de germinação foi calculado de acordo com Handro (1969) e Ferreira (1977). Em alguns casos os dados foram transformados em arc sen.$\sqrt{x}$ ou . $\sqrt{y_{1 \rightarrow j}+1 / 2}$ para as comparações, quando não havia homogeneidade ou coeficiente de variação era alto ou ainda quando havia valores zero na distribuição.

\section{Resultados}

O tempo médio de germinação é um bom índice para avaliar-se a rapidez de ocupação 
de uma espécie em um determinado nicho ou território. Neste trabalho, quanto ao tempo médio de germinação (Tab. 1)as sementes foram classificadas em: rápidas ( tempo médio $<5$ dias); intermediárias (tempo médio > $5<10$ dias); lentas ( tempo médio > 10 dias ). Observa-se na Tabela 1 , que há um grupo de espécies (B,trimera; E.mollis; M.cordifolia: S.casarettoi) em que o tempo médio de germinação a $20^{\circ} \mathrm{C}$ é ,mais longo significativamente do que a 25 ou $30^{\circ} \mathrm{C}$.

Quando a germinação ocorreu em temperaturas alternadas (Tab. 2), com regime de 8 horas diárias de luz, os percentuais de germinação foram semelhantes em vários pares de temperaturas, a excessão de T.minuta em que a presença de uma temperatura acima de $25^{\circ} \mathrm{C}$ foi prejudicial. Para M.cordifolia a presença de luz ou não, foi mais importante do que a variação de temperatura (Tab. 2 e 3).

Quanto a presença de luz, as espécies estudadas podem ser divididas em 4 grupos (Tab. 3): 1- As afotoblásticas (S.campestre); 2 - as fotoblásticas positivas relativas (E.alba; E.mollis; S.oxyphyllus; S.selloi; S. casarettoi e Vernonia nudiflora). Este termo refere-se a percentuais de germinação significativamente mais altos na luz, mas sem no entanto atingirem mais que o dobro do regime do escuro. 3-As fotoblásticas positivas, (B.trimera; E.laevigatum; M.cordifolia; S.heterotrichius e T.praestens) quando o percentual de germinação na luz foi mais que o dobro do percentual no escuro. 4Fotoblástica negativa relativa (T.minuta) quando germinou melhor no escuro.

Somente quatro espécies (E.mollis; S.casarettoi; T,minuta e V.nudiflora) tiveram percentual de germinação acima de 50\% ( Tab. 3). Em algumas espécies (B.trimera; M.cordifolia; S.heterotrichius; T.praestens) o percentual máximo de germinação não ultrapassou $35 \%$. Em todos os casos, houve seleção prévia dos aquênios, tendo sido descartados os visivelmente mal formados.
Há espécies ( Fig. 1; 2 e 3), em que o percentual de germinação não variou nas três temperaturas constantes usadas ( B.trimera, E.mollis; E.laevigatum; S.oxyphyllus). Nas demais espécies a $30^{\circ} \mathrm{C}$, o percentual de germinação foi significativamente menor (Fig. 1; 2 e 3). Finalmente em S.heterotrichius, S.selloi e T.minuta a $20^{\circ} \mathrm{C}$ o percentual de germinação foi maior do que 25 ou $30^{\circ} \mathrm{C}$ ( Fig 2 e 3).

Espécie por espécie, os diásporos das Asteraceae, quanto à germinação, tiveram o seguinte comportamento:

Baccharis trimera: Tempo médio mais curto a $25^{\circ} \mathrm{C}$, mas pouco diferente de a 20 ou $30^{\circ} \mathrm{C}$. Menos de 5 dias para germinação média portanto germinação rápida. Baixos percentuais de germinação, abaixo de $20 \%$ em qualquer temperatura de 20 a $30^{\circ} \mathrm{C}$. Fotoblastismo: positivo.

Eclipta alba: Tempo médio mais curto a $25^{\circ} \mathrm{C}$, bem mais longo a 20 ou $30^{\circ} \mathrm{C}$. Menos de 5 dias de tempo médio portanto germinação rápida. Alternância de temperatura necessária para promover germinação (86 a 93\%), em temperatura constante germinação máxima a $30^{\circ} \mathrm{C}$ foi de $63 \%$. Em temperaturas mais baixas germina menos, $20^{\circ} \mathrm{C}$ aproximadamente $15 \%$. Fotoblastismo: positivo relativo. Elephantopus mollis: tempo médio curtíssimo a 25 ou $30^{\circ} \mathrm{C}$. Germinação rápida. Não importa a temperatura, a percentagem de germinação foi entre 50 e $60 \%$. Fotoblastismo: positivo relativo.

Eupatorium laevigatum: tempo médio de germinação intermediário, não importando a temperatura. Percentual de germinação em torno de $30 \%$ à temperatura constante ou alternada. Fotoblastismo: positivo absoluto, não germinam no escuro.

Mikania cordifolia: tempo médio para germinação intermediário, não importando a temperatura. Percentual de germinação em torno de $30 \%$ não importando se as temperaturas foram alternadas ou constantes. A $30^{\circ} \mathrm{C}$ 
Tabela 1 - Tempo médio (em dias) de germinação em três temperaturas constantes e fotoperíodo de $12 \mathrm{~h} / \mathrm{luz},+/-4 \mathrm{Wm}^{-2}$

\begin{tabular}{llcc}
\hline Espécies & \multicolumn{3}{c}{ Temperaturas } \\
\cline { 2 - 4 } & $\mathbf{2 0}^{\circ} \mathbf{C}$ & $\mathbf{2 5}^{\circ} \mathbf{C}$ & $\mathbf{3 0}^{\circ} \mathbf{C}$ \\
\hline Baccharis trimera & $5,11(\mathrm{~b})$ & $4,43(\mathrm{a})$ & $4,59(\mathrm{ab})$ \\
Eclipta alba & $10,09(\mathrm{c})$ & $4,82(\mathrm{a})$ & $7,55(\mathrm{~b})$ \\
Elephantopus mollis & $3,43(\mathrm{~b})$ & $2,62(\mathrm{a})$ & $2,42(\mathrm{a})$ \\
Eupatorium laevigatum & $10,31(\mathrm{a})$ & $8,58(\mathrm{a})$ & $9,82(\mathrm{a})$ \\
Mikania cordifolia & $9,20(\mathrm{~b})$ & $8,30(\mathrm{ab})$ & $7,80(\mathrm{a})$ \\
Senecio heterotrichius & $15,45(\mathrm{a})$ & $14,56(\mathrm{a})$ & $15,25(\mathrm{a})$ \\
Senecio oxyphyllus & $12,40(\mathrm{a})$ & $10,75(\mathrm{a})$ & $10,32(\mathrm{a})$ \\
Senecio selloi & $12,30(\mathrm{a})$ & $13,50(\mathrm{a})$ & $13,40(\mathrm{a})$ \\
Stenachaenium campestre & $3,26(\mathrm{a})$ & $3,58(\mathrm{a})$ & $8,72(\mathrm{~b})$ \\
Symphyopapus casarettoi & $7,87(\mathrm{~b})$ & $6,73(\mathrm{a})$ & $6,78(\mathrm{a})$ \\
Tagetes minuta & $7,25(\mathrm{a})$ & $9,00(\mathrm{~b})$ & - \\
Trixis praestans & $11,40(\mathrm{a})$ & $12,60(\mathrm{a})$ & $12,4(\mathrm{a})$ \\
Vernonia nudiflora & $3,51(\mathrm{a})$ & $3,12(\mathrm{a})$ & $3,48(\mathrm{a})$ \\
\hline
\end{tabular}

Tempos médios seguidos por mesma letra , nas linhas, não diferem entre si com probabilidade de 95\%, utilizando-se intervalos de confiança calculado segundo Handro (1969) e Ferreira (1977).

Tabela 2 - Percentual de germinação das sementes submetidas a temperaturas alternadas no fotoperíodo de $\left.8 \mathrm{~h} / \mathrm{luz}_{(4 \mathrm{Wm}}{ }^{-2}\right)$, submetidas à temperatura mais alta.

\begin{tabular}{|c|c|c|c|c|}
\hline \multirow[t]{2}{*}{ Espécies } & \multicolumn{4}{|c|}{ Temperaturas } \\
\hline & $20 / 10$ & $25 / 15$ & $30 / 20$ & $35 / 25$ \\
\hline Eclipta alba & 90 (a) & $92(\mathrm{a})$ & $93(\mathrm{a})$ & $86(a)$ \\
\hline Eupatorium laevigatum & $36(a)$ & 34 (a) & 35 (a) & 33 (a) \\
\hline Mikania cordifolia & $21,6(a)$ & $25,6(a)$ & $34,6(a)$ & 28,5 (a) \\
\hline Stenachaenium campestre & $82(a)$ & $83(\mathrm{a})$ & 79 (a) & 34 (b) \\
\hline Tagetes minuta & $84(a b)$ & 100 (a) & $57(\mathrm{bc})$ & $68(\mathrm{c})$ \\
\hline
\end{tabular}

Médias seguidas de mesma letra, na linha, não diferem significativamente pelo teste da Duncan ( $\mathrm{p}<5 \%$.

Tabela 3 - Porcentagem média de germinação na luz e no escuro de 13 espécies de Asteraceae.

\begin{tabular}{lllc}
\hline \multirow{2}{*}{ Espécies } & \multicolumn{2}{c}{ Regime de luz } & \multirow{2}{*}{ Fotoblastismo } \\
\cline { 2 - 3 } & Luz & Escuro & \\
\hline Baccharis trimera & $33,1(\mathrm{a})$ & $2,4(\mathrm{~b})$ & + \\
Eclipta alba & $41,2(\mathrm{a})$ & $26,0(\mathrm{~b})$ & $+\mathrm{r}$ \\
Elephantopus mollis & $68,9(\mathrm{a})$ & $36,0(\mathrm{~b})$ & $+\mathrm{r}$ \\
Eupatorium laevigatum & $26,0(\mathrm{a})$ & $0,0(\mathrm{~b})$ & + \\
Mikania cordifolia & $34,3(\mathrm{a})$ & $6,0(\mathrm{~b})$ & + \\
Senecio heterotrichius & $29,2(\mathrm{a})$ & $10,8(\mathrm{~b})$ & + \\
Senecio oxyphyllus & $46,9(\mathrm{a})$ & $23,8(\mathrm{~b})$ & $+\mathrm{r}$ \\
Senecio selloi & $49,5(\mathrm{a})$ & $28,5(\mathrm{~b})$ & $+\mathrm{r}$ \\
Stenachaenium campestre & $45,2(\mathrm{a})$ & $64,6(\mathrm{a})$ & 0 \\
Symphyopapus casarettoi & $66,0(\mathrm{a})$ & $26,2(\mathrm{~b})$ & $+\mathrm{r}$ \\
Tagetes minuta & $62,0(\mathrm{~b})$ & $83,5(\mathrm{a})$ & $-\mathrm{r}$ \\
Trixis praestans & $22,2(\mathrm{a})$ & $7,5(\mathrm{~b})$ & + \\
Vernonia nudiflora & $72,4(\mathrm{a})$ & $59,3(\mathrm{~b})$ & $+\mathrm{r}$ \\
\hline
\end{tabular}

Médias seguidas de mesma letra, na linha, não diferem significativamente pelo teste da Duncan $(\mathrm{p}<5 \%)$.

Fotoblastismo positivo $(+)$, relativo $(+r)$, negativo relativo $(-r)$ e neutro $(0)$. 
a germinação é prejudicada. Fotoblastismo: positivo.

Senecio heterotrichius: tempo médio lento, nas temperaturas estudadas. Temperatura mais baixa $\left(20^{\circ} \mathrm{C}\right)$ favorável a germinação. Fotoblastimo: positivo.

Senecio oxyphyllus: tempo médio de germinação é lento, não importando a temperatura. Tendência a germinar mais a $20^{\circ} \mathrm{C}$, mas em percentuais que não passam de $40 \%$. Fotoblastismo: positivo relativo.

Senecio selloi: tempo médio de germinação é lento, nas temperaturas estudadas. A $20^{\circ} \mathrm{C}$ melhor temperatura para germinação, com mais 50\%. Fotoblastimo: positivo relativo.

Stenachaenium campestre: tempo médio a 20 ou $25^{\circ} \mathrm{C}$ é rápido. Alternância de temperaturas ou temperatura constante, germinação em torno de $80 \%$, desde que uma das temperaturas seja abaixo de $30^{\circ} \mathrm{C}$.Fotoblastismo: neutro, sementes afotoblásticas.

Symphyopappus casarettoi: tempo médio intermediário, não importando a temperatura entre 20 e $30^{\circ} \mathrm{C}$. Germina melhor a $20^{\circ} \mathrm{C}$, mais de $60 \%$, do que a $30^{\circ} \mathrm{C}$; menos de $30 \%$. Fotoblastismo: positivo.

Tagetes minuta: Tempo médio de germinação intermediário. Sob alternância de temperatura pode chegar a $100 \%$ de germinação $\left(25 / 15^{\circ} \mathrm{C}\right)$, ou se a temperatura for constante em torno de $20^{\circ} \mathrm{C}$. Fotoblastismo: negativo relativo.

Trixis praestens: tempo médio de germinação é lento, nas temperaturas estudadas. Percentuais baixos de germinação, não passando $20 \%$ a $20^{\circ} \mathrm{C}$, sendo mais baixos ainda a $30^{\circ} \mathrm{C}$, em torno de $10 \%$. Fotoblastismo: positivo.

Vernonia nudiflora: tempo médio de germinação rápido, nas temperaturas testadas. Germinação de mais $70 \%$ a 20 ou $25^{\circ} \mathrm{C}$. Fotoblastismo: positivo relativo.

\section{Discussão}

O estudo da germinação de 13 espécies de Asteraceae comuns no estado do Rio Grande do Sul em ambientes abertos, permite fazer algumas especulações sobre a possível ocupação do facies campestre por estas espécies. Muitas compostas comportam-se como invasoras, sendo algumas delas assim consideradas (Kissmann \& Groth, 1992).

A presença ou ausência de luz, combinada com diferentes temperaturas, são fatores ambientais dos mais comuns como agentes desencadeadores da germinação. Estes fatores em conjunto com a água, especialmente nos microsítios do solo, regulam a germinação (Bai \& Romo, 1995).

Pode haver interação entre luz e temperatura promovendo a germinação de sementes (Taylorson \& Hendricks, 1972), fenômeno que é comum entre as plantas invasoras. No presente estudo foi observado interação de luz e temperatura apenas em poucas espécies como S.selloi, T.praestans e V.nudiflora, sendo que para a maioria das espécies não há interação entre os dois fatores (dados não apresentados), resultados semelhantes foram observados para Artemisia frigida (Bai et al., 1995).

A luz, entendida como a quantidade de energia radiante a $660 \mathrm{~nm}$ suficiente para tornar ativo o mecanismo do fitocromo (Kendrick, 1976), promove a germinação de sementes de Asteraceae em 11 das 13 espécies estudadas e o fenômeno foi observado para várias espécies desta família por diversos autores (Soriano et. al., 1963; Valio et al.,1972; Baskin \& Baskin, 1979; 1985; 1988; Brown \& Forsyth, 1981; Martinez et al., 1992; Reddy \& Singh, 1992; Garcia \& Sharif, 1995; Bell et al. ,1999). Foram usadas sementes recém colhidas, pois o armazenamento pode alterar os requerimentos de luz e temperatura para a germinação de muitas espécies de compostas (Baskin \& 


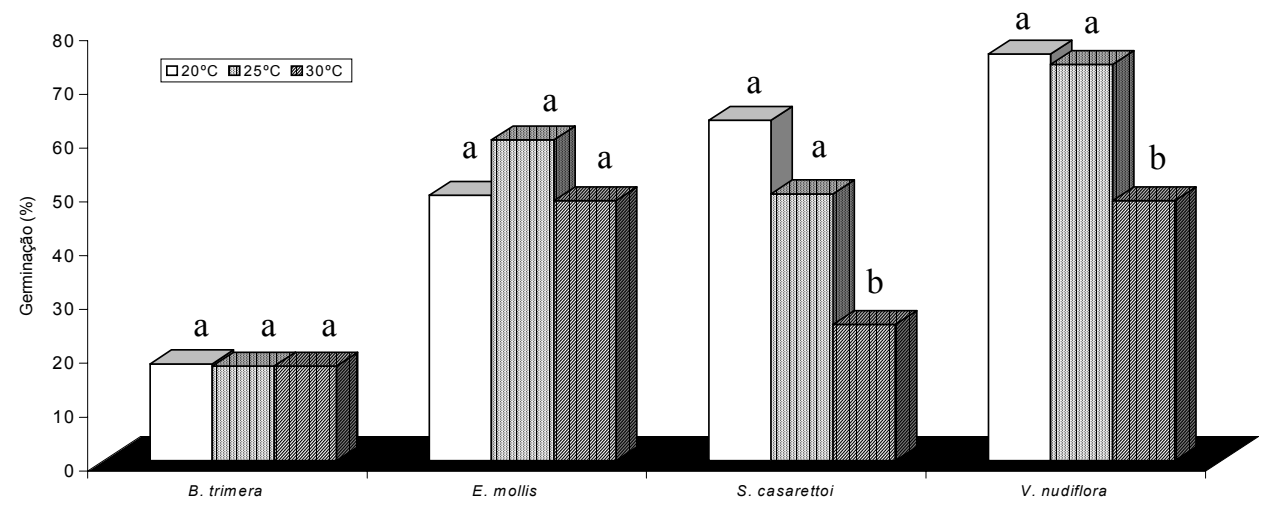

Figura 1: Porcentagem de sementes germinadas de Bacharis trimera, Elephantopus mollis, Symphyopapus casarettoi e Vernonia nudiflora

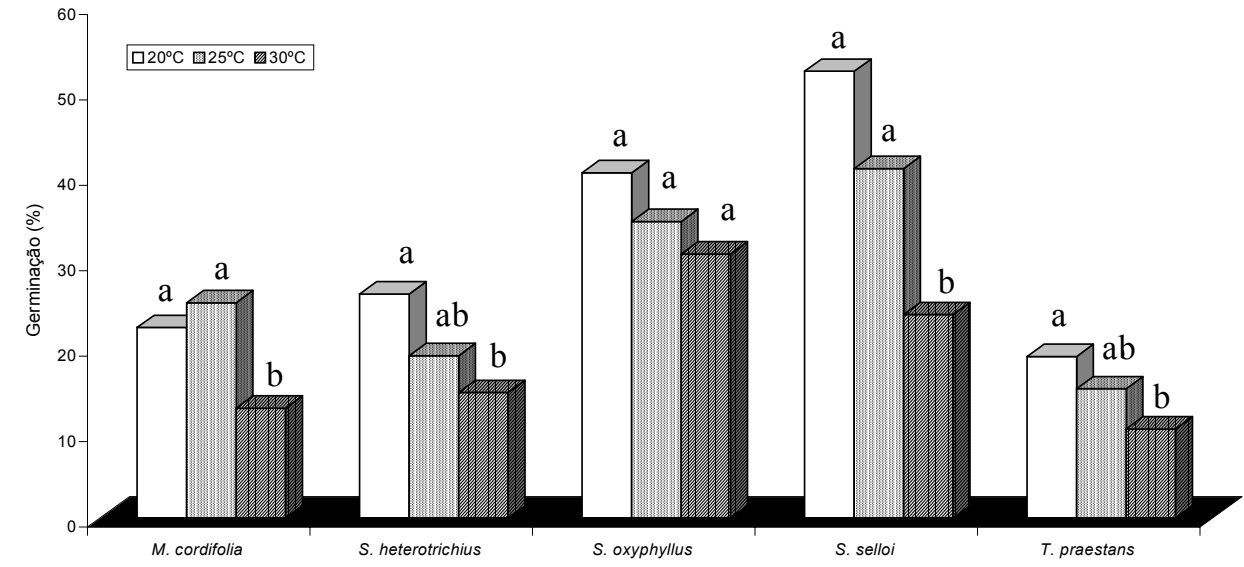

Figura 2: Porcentagem de sementes germinadas de Mikania cordifolia, Senecio heterotrichius, Senecio oxyphyllus, Senecio selloi e Trixis praestans

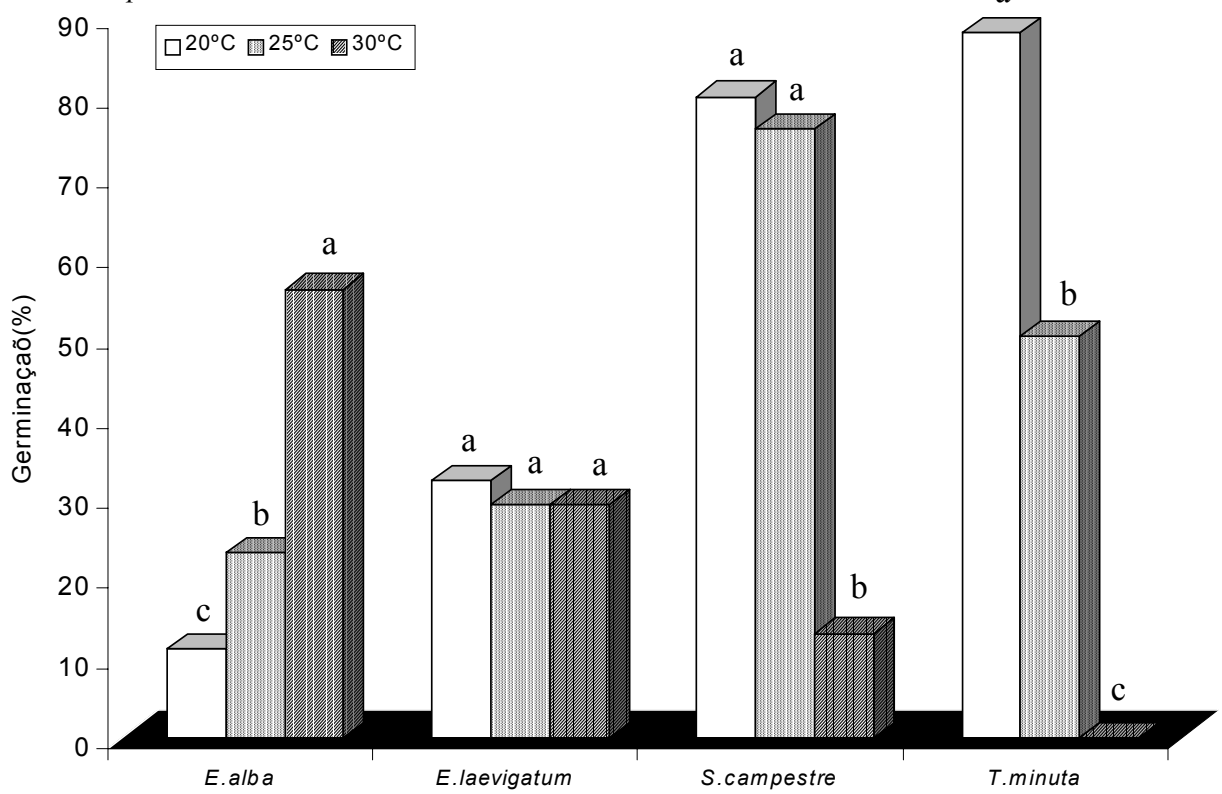

Figura 3: Porcentagem de sementes germinadas de Eclipta alba, Eupatorium laevigatum, Stenachaenium campestre e Tagetes minuta 
Baskin, 1979; Globerson,1981; Thanos \& Georghiou, 1988; Choi et al., 1989; Baskin et al.,1993; Bai et al., 1995; Carnelossi et al., 1995).

Outros dois fatores muito importantes para o comportamento germinativo de várias espécies entre as de Asteraceae, dizem respeito a: 1- História da planta mãe e dos estresses sofridos durante o desenvolvimento das sementes. Isto pode alterar drasticamente o comportamento germinativo dos aquênios (Perez-Garcia, 1993; Bai \& Romo, 1994); 2- Dimorfismo ou polimorfismo dos aquênios. Este fenômeno é comum nos capítulos de Asteraceae (McEvoy, 1984; Tanowitz et al., 1987; Venable et al., 1987; Imbert et al., 1996;; Rocha, 1996; Amaral \& Takaki, 1998). Estas variações podem não ser só quanto a forma, mas também quanto à pigmentação dos aquênios (Randi \& Felippe, 1980). No entanto, não foram utilizados estes critérios, que podem eventualmente ocorrer, para a separação dos aquênios neste estudo. Também não houve controle das condições de desenvolvimento das sementes na planta-mãe, já que as coletas foram expeditas e realizadas numa única ocasião para cada espécie.

Variações de temperatura podem aumentar a germinabilidade de sementes de várias compostas ( Drew \& Brocklehurst, 1984; Ghersa et al., 1992) ou de gramíneas de ambientes abertos (Carmona et al, 1998). Neste estudo para E.alba e para S.campestre isto ficou bastante evidente (tabela 2). Para E.alba temperatura mais alta $\left(30^{\circ} \mathrm{C}\right)$ promoveu a germinação, resultados similares aos encontrados por Altom e Murray (1996), embora o tempo médio de germinação tenha sido mais baixo a $25^{\circ} \mathrm{C}$ (Tab. 1)

Tagetes minuta foi a única espécie que se mostrou fotoblástica negativa relativa - a germinabilidade na luz foi mais que a metade daquela verificada no escuro em percentual, mas significativamente inferior-, embora este resultado discorde com aqueles obtidos por outros autores (Soriano et al., 1963; Forsyth \& Van Staden, 1983). Mudanças na temperatura ou regime de luz podem promover a germinação da espécie (Forsyth \& Van Staden, 1983), havendo termo-inibição à temperaturas mais altas (Drewes \& Van Staden, 1990). Isto explicaria, porque obteve-se germinação mais alta a temperatura $20^{\circ} \mathrm{C}$. Também não se pode descartar que o comportamento fotoblástico negativo observado seja devido a historia da planta-mãe ou ecotipo diferente dos estudados pelos outros autores.

Para o gênero Senecio foram examinadas três espécies S.heterotrichius, S.oxyphyllus e S.selloi, todas com sementes fotoblásticas positivas, com tempo médio de germinação lento e com tendência à germinabilidade maior a $20^{\circ} \mathrm{C}$ ( Fig. 2). Portanto as três espécies apresentam requerimentos semelhantes à germinação. Para S.vulgaris também foi encontrado que a germinação foi favorecida a $20^{\circ} \mathrm{C}$ (Hilton, 1983; Ren \& Abbott, 1991), sendo que para S.crassifolius a 10 ou $15^{\circ} \mathrm{C}$ ou ainda alternância entre $10 \mathrm{e}$ $20^{\circ} \mathrm{C}$ houve um percentual mais alto de germinação (Cordazzo \& Souza, 1993). Tratamentos pré-germinativos de frio aumentaram a germinabilidade de S.serra, especialmente em temperaturas alternadas, ainda que a germinação seja lenta (McDonough, 1974). Embora o gênero Senecio seja um dos maiores nas Asteraceae, várias espécies tem comportamento germinativo similar. $\mathrm{Na}$ maioria são fotoblásticas positivas, lentas para germinar, germinando em percentuais mais altos quando a temperatura é em torno de $20^{\circ} \mathrm{C}$ ou mais baixa, ou pelos menos quando um dos pares o é. Este comportamento possibilita a estas espécies serem as que se instalam logo após o inverno e os mecanismos comportamentais que permitem sua agressividade em termos de domínio em muitas paisagens abertas observadas nas re- 
giões semi-temperadas, devem ser eficientes pós-instalação das plantas.

O baixo poder germinativo foi encontrado para E.laevigatum quer em temperaturas constantes (Fig. 3) quer em temperaturas alternadas (tabela 2) indo pouco alem dos $30 \%$ de germinação, sendo fotoblásticas positivas como E.vauthierianum (Maluf \& Wizentier, 1998) e E.pauciflorum, embora esta última espécie tenha sido de rápida germinação (Figueiredo, 1975).

Baixa germinação pode ser um problema importante não só sob o ponto de vista ecológico, mas particularmente, quando se deseja exploração econômica de uma composta (Afolayan et al, 1997). No presente trabalho, incluem-se algumas Asteraceae de importância como plantas medicinais (B.trimera; M.cordifolia e E.laevigatum) as quais tiveram baixos percentuais de germinação ( Tab. 2 e Fig.s 1; 2; 3). Este problema necessitaria de uma abordagem futura.

Há vários substratos que são usados para testes em germinação de sementes, todos com algumas vantagens ou desvantagens. Em placas de Petri ou caixas gerbox, papel de filtro ou "germitest" são comumente usados, apresentando vantagens como fácil observação da emergência da radícula, portanto germinação das sementes, sem manipulações especiais; embora facilmente possam ser inundadas com acréscimo excessivo de solução umidificante. Também facilmente podem secar, particularmente nos testes a temperaturas mais altas. Além disto, deve ser papel de boa qualidade, para evitar problemas de toxidez (Ferreira et al., 1994). Rolos de papel são aconselhados pela RAS (Brasil, 1992), tendo a vantagem de dificilmente secar, mas necessita de maior manuseio na instalação e observações, além de ser impossível seu uso para verificação de fotoblastimo na germinação. Areia , que não apresenta estes inconvenientes, torna mais difícil a observação expedita de sementes em germinação, dificultando bastante quando os diásporos são pequenos, como comumente observa-se entre as Asteraceae. O uso de ágar de boa qualidade na concentração de $1 \%$, evita os problemas de eventual seca, inundação, transparência ou dificuldades de visualização, sendo bastante vantajoso, já tendo sido usado anteriormente (Rosa \& Ferreira, 1998; 1999).

Cada semente tem seu requerimento para germinação. Pode ser que numa população de uma espécie haja um mesmo requerimento para um grande percentual de suas sementes. Em geral, há um sinergismo, sendo um fator limitante acompanhado de outros limitantes adjuntos. Quando ocorrem condições ambientais favoráveis excepcionais, há um sincronismo de rompimento combinado a superação dos limitantes e uma grande explosão de geminação daquela espécie pode ocorrer, assim como de outras que tenham semelhantes requerimentos.

\section{Agradecimentos}

Aos professores Dr Nelson I. Matzembacher e MSc Mara R.Ritter pela identificação botânica das espécies usadas. Ao Dr. João Riboldi por sugestões quanto ao tratamento estatístico. Ao professor Dr. Arthur G. Fett-Neto por correções no inglês. A inúmeros alunos da disciplina de Fisiologia da Germinação de Sementes que colaboraram reetestando muito dos resultados alcançados. A FAPERGS e PROPESQ/UFRGS por auxílios que ajudaram na manutenção do laboratório de Fisiologia Vegetal, e ao CNPq e CAPES pelas bolsas outorgadas.

\section{Referências bibliográficas}

Afolayan, A.J.; Meyer, J.J.M. \& Leenwner, D.V. 1997. Germination in Helichrysum aureonitens (Asteraceae): effects of temperature, light , gibberellic acid, scarification and smoke extract. South African Journal of Botany 63(1): 22-24. 
Altom, J.V. \& Murray, D.S. 1996. Factors affecting eclipta (Eclipta prostrata) seed germination. Weed Technology 10: 727-731.

Amaral, A. \& Takaki, M. 1998. Achene dimorphism in Bidens pilosa L. (Asteraceae) as determinad by germination test. Brazilian Archives of Biology and Technology 41(1): 11-16.

Aranha, C.; Leitão-Filho, H.F. \& Yahn, C.A. 1987. Sistemática de Plantas Invasoras. Inst. Camp.Ens.Agric., Campinas.

Bai, Y. \& Romo, J.T. 1994. Germination of previously buried seeds of fringed sage (Artemisia frigia) Weed Science 42: 390-397.

Bai, Y. \& Romo, J.T. 1995. Seedling emergence of Artemisia frigida in relation to hydrationdehydration cycles and seedbed characteristics. Journal of Arid Environments 30: 57-65.

Bai, Y.; Romo, J.T. \& Young, J.L. 1995. Influences of temperature, light and water stress on germination of fringed sage ( Artemisia frigida). Weed Science 43: 219-225.

Baskin, C.C. \& Baskin, J.M. 1988. Germination ecophysiology of herbaceous plant species in a temperate region. American Journal of Botany 75(2): 286-305.

Baskin, C.C. \& Baskin, J.M. 1998. Seeds: ecology, biogeography and evolution of dormancy and germination. Acad. Press, San Diego.

Baskin, C.C. : Baskin, J.M. \& Leck, M.A. 1993. Afterripening pattern during cold stratification of achenes of ten perennial Asteraceae from Eastern North America and evolutionary implication. Plant Species Biology 8: 61-65.

Baskin, J.M. \& Baskin, C.C. 1979. The germination strategy of oldfield aster (Aster pilosus). American Journal of Botany 66(1): 1-5.

Baskin, J.M. \& Baskin, C.C. 1985. The light requirement for germination of Aster pilosus seeds: temporal aspects and ecological consequences. Journal of Ecology 73: 765-773.

Bell, D.T.: King, L.A. \& Plummer, J.A. 1999. Ecophysiological effects of light quality and nitrate on seed germination in species from western Australia. Australian Journal of Ecology. 24: 2-10.

Boldrini, I.I. \& Eggers, L. 1996. Vegetação campestre do sul do Brasil: dinâmica de espécies à exclusão do gado. Acta Botanica Brasilica 10(1): 37-50.

Brasil.1992. Regras de análise de sementes. Ministério da Agricultura e da Reforma Agrária. Brasília
Brown, N.A.C. \& Forsyth, C. 1981. Demonstration of the reversible photoreaction controlling germination using achenes of Bidens pilosa L. (blackjack). South African Journal of Science 77: 239.

Carmona, R.; Martins, C.R. \& Fávaro, A.P. 1998. Fatores que afetam a germinação de sementes de gramíneas nativas do cerrado. Revista Brasileira de Sementes 20(1): 16-22.

Carnelossi, M.A.G.; Lamourier, L. \& Ranal, M.A. 1995. Efeito da luz, hipoclorito de sodio, escarificação e estratificação na germinação de sementes de alface (Lactuca sativa L.) C.V. Marobá e moreninha-deUberlândia. Pesquisa Agropecuaria Brasileira 30(6): 779-787.

Cheng, H.H. 1992. A conceptual framework for assessing allelochemicals in the soil environment. Pa 21-29. In S.J.H. Rizvi \& V.Rizvi(eds) Allelopathy: Basic and applied aspects. Chapman Hall, London.

Choi, K.S.; Watanabe,M. \& Furuya,M. 1989. Effects of long-term storage on phytochrome0mediated germination in lettuce seeds. Botanical Magazine Tokyo 102: 181-191.

Cordazzo, C.V. \& Souza, H.Z. 1993. Germinação de Senecio crassiflorus (Compositae) Revista Brasileira de Biologia 53(1): 81-86.

Drew, R.L.K. \& Brocklehurst, P.A. 1984. Investigations on the control of lettuce seeds germination at high temperatures. Journal of Experimental Botany 35(156): 986-993.

Drewes, F.E. \& Van Staden, J. 1990. Germination of Tagetes minuta 1. II. Role of gibberelins. Plant Growth Regulation 9: 285-291.

Egley, G.H. 1995. Seed germinatrion in soil: dormancy cycle. Pp. 529-543. In J.Kigel \& G.Galili. Seed development and germination. Marcel Decker, New York.

Esashi, Y. \& Tsukada, Y. 1978. Thermoperiodism in cocklebur seed germination. Plant Physiology 61: 437-441.

Ferreira, A.G.; Borghetti, F.: Schwamback, L. \& Silveira, T.S. 1994. Efeito do substrato e $\mathrm{pH}$ no desenvolvimento inicial de plantas. Caderno de Pesquisa serie Botanica 6(1): 13-23.

Ferreira, A.G. 1977. Araucaria angustifolia (Bert.)0.Ktze.: germinação da semente e desenvolvimento da plântula. Tese de doutoramento. USP. São Paulo.

Figueiredo, R.R. 1975. Notas preliminares sobre a germinação e ocorrência de derivados cumarínicos em aquênios de Eupatorium pauciflorum H.B.K. (Compositae). Hoehnea 5: 47-57. 
Forsyth, C. \& Van Staden, J. 1983. Germination of Tagetes minuta L. I. Temperature effects. Annals of Botany 52: 659-666.

Frankland, B. \& Taylorson, R. 1983. Light control of seed germination. Pp. 428-456. In W.Shropshine \& H.Mohr (eds). Photomorphogenesis vol $16^{\mathrm{A}}$. Springer-Verlag, Berlin.

Garcia, Q.S. \& Sharif, R.R. 1995. Germinação e dormência em aquênios de Acanthospermum hispidum DC., uma espécie invasora. Revista Brasileira de Botânica 18(1): 17-25.

Gavilanes, M.L. \& D’Angieri-Filho, C.N. 1991. Flórula ruderal da cidade de Lavras, MG. Acta Botanica Brasilica 5(2) 77-88.

Ghersa, C.M.; Benech Arnold, R.L. \& Martinez-Ghersa, M.A. 1992. The role of fluctuating temperatures in germination and establishment of Sorghum hapelense. Regulation of germination at increasing depths. Functional Ecology 6: 460468.

Globerson, D. 1981. Germination and dormancy in immature and fresh-mature lettuce seeds. Annals of Botany 48: 639-643.

Handro, W. 1969. Contribuição ao estudo da unidade de dispersão e da plântula de Andira humilis Mart. Ex Benth (Leguminosae - Lotoideae) Boletim Faculdade de Filosofia Ciências e Letras da Universidade de São Paulo. 349 Botânica, 27: 1-189.

Hilton, J.R. 1983. The influence of light on the germination of Senecio vulgaris L. The New Phytologist 94: 29-37.

Imbert, E.; Escarré,J. \& Lepart, J. 1996. Achene dimophism and among-population variation in Crepis sancta (Asteraceae). International Journal of Plant Science 157(3): 309-315.

Kendrick, R.E. 1976. Photocontroll of seed germination. Science Progress, 63: 347-367.

Kissmann, K.G. \& Groth, D. 1992. Plantas Infestantes e Nocivas . vol 2. Basf. Linburgerhof.

Labouriau, L.F.G. \& Costa, J.A.F. 1976. Objetivos e instalações básicas de um laboratório de Fisiologia Vegetal. Academia Brasileira de Ciencias, Rio de Janeiro.

Little, T.M. \& Jackson Hills, F. 1978. Agricultural experimentations: Design and Analysis. J.Wiley $\&$ Sons, New York.

Maluf, A.M. \& Wizentier, B. 1998. Aspectos fenológicos e germinação de sementes de quatro populações de Eupatorium vauthierianum DC (Asteraceae). Revista Brasileira de Botanica 21(3): 247-257.
Martinez, M.L. ; Valverde, T. \& Moreno-Casasola, P. 1992. Germination response to temperature, salinity, light and depth of sowing of ten tropical dune species. Oecologia 92: 343-353.

McDonough, W.T. 1974. Effect of temperature pretreatment of achenes of Senecio serra on germination during stratification. Canadian Journal of Botany 52: 1985-1987.

McEvoy, P.B. 1984. Dormancy and dispersal in dimorphic achenes of tansey ragwort, Senecio jacobaea L. (Compositae) Oecologia 61: 160168 .

Perez-Garcia, F. 1993. Effect of the origin of the cypsela on germination of Onopordum acanthium L.(Asteracae) Seed Science and Technology 21: 187-195.

Randi, A.M. \& Felippe, G.M. 1980. Detecção de esteviosídeo e substâncias giberelinicas nos aquênios de Stevia rebaudiana: efeito de esteviosídeo em germinação. Revista Brasileira de Botânica 3: 55-58.

Reddy, K.N. \& Singh, M. 1992. Germination and emergence of hairy beggarticks (Bidens pilosa). Weed Science 40: 195-199.

Ren, Z. \& Abbott, R.J. 1991. Seed dormancy in mediterranean Senecio vulgaris L The New Phytologist 117 : 673-678.

Rocha, O.J. 1996. The effects of achene heteromorphism on the dispersal capacity of Bidens pilosa L. International Journal of Plant Science 157(3): 316-322.

Rosa, S.G.T. \& Ferreira, A.G. 1998. Germinação de sementes de espécies medicinais do Rio Grande do Sul: Bromelia antiacantha Bert.; Cuphea carthagenesis (Jacq.)Macbride e Talinum patens (Jacq.)Willdenov. Acta Botanica Brasilica 12(3): 515-522.Suplemento.

Rosa, S.G.T. \& Ferreira, A.G. 1999. Germination of medicinal plant: Smilax campestris Griseb. (salsaparrilha). Acta Horticulturae 502: 105-111.

Schupp, E.W. \& Fuentes, M. 1995. Spatiall patterns of seed dispersal and the unification of plant populations ecology. Ecoscience 2(3): 267-275.

Smith, H. \& Morgan, D.C. 1983. The function of phytochrome in nature.Pp 412-517. In W.Shropshire \& M.Mohr (eds). Photomorphogenesis vol.16B. Springer Verlag, Berlin.

Sokal, R.R. \& Rohlf, F.J. 1981. Biometry. W.H.Freenan, San Francisco.

Soriano, A.; Eilberg, B.A. \& Slabnik, E. 1963. Comportamiento de la germinacion de semillas 
de malezas anuales. Revista de Investigaciones Agricolas 17(4): 447-464.

Tanowitz, B.D.; Salopek, P.F. \& Mahall, B.E. 1987. Differential germination of ray and disc achenes in Hemizonia increacens (Asteraceae) American Journal of Botany 74(3): 303-312.

Taylorson, R. \& Hendricks, S.B. 1972. Interactions of light and a temperature shift on seed germination. Plant Physiology 49: 127-130.

Thanos, C.A. \& Georghiou, K. 1988. On the mechanism of skotodormancy induction in grand rapids lettuce (Lactuca sativa L.) seeds. Journal of Plant Physiology 133: 580-584.

Thompson, P.A. 1974. Effects of fluctuating temperatures on germination. Journal of Experimental Botany 25(84): 164-175.

Thompson, K. \& Grime, J.P. 1983. A comparative study of germination responses to diurnallyflutuating temperatures. Journal Applied Ecology. 20: 141-156.

Toledo, J.R.; Rincón, E. \& Vasquez-Yanes, C. 1990. A light quality gradiant for the study of red: farred ratios on seed germination. Seed Science \& Technology. 18: 277-282.

Toole, E,H.: Hendricks, S.B.; Borthwick, H. \& Toole, V.K. 1956. Physiology of seed germination. Annual Review of Plant Physiology. 7: 299-324.

Valio, I.F.M. : Kirszenzaft, S.L. \& Rocha, R.S. 1972. Germination of achenes of Bidens pilosa L. I. Effect of light of different wavelenghts . The New Phytologist 71: 677-682.

Venable, D.L. ; Burquez, A.; Corral, G.; Morales, E. \& Espinosa, F. 1987. The ecology of seed heteromorphism in Heterosperma pinnatum in Central Mexico. Ecology 68(1): 65-76. 